\title{
Form and Freedom: \\ The Kantian Ethos of Musical Formalism
}

\author{
Hanne Appelqvist
}

\begin{abstract}
AвSTRACT Musical formalism is often portrayed as the enemy of artistic freedom. Its main representative, Eduard Hanslick, is seen as a purist who, by emphasizing musical rules, aims at restricting music criticism and even musical practices themselves. It may also seem that formalism is depriving music of its ability to have moral significance, as the semantic connection to the extramusical is denied by the formalistic view. In my paper, I defend formalism by placing Hanslick's argument in a Kantian framework. It is not hard to find Kantian elements in Hanslick's work, such as his emphasis on the contemplative and disinterested nature of the aesthetic judgment, the nonconceptuality of music's content, and his insistence that "beauty has no purpose." I argue further that Hanslick's formalism is in fact motivated by and manifests the Kantian conception of freedom as self-legislation. Thus understood, the kind of moral significance music may have rests upon its own autonomous rules. KEYWORDS Hanslick, Kant, formalism, musical understanding, autonomy of music
\end{abstract}

\section{Introduction}

Musical formalism is not a popular view. At least not in the original sense of the term which refers to Eduard Hanslick's claim that the content of music is nothing but "tonally moving forms."1 In the literature, a typical way of addressing Hanslick is to portray him as a "narrow-minded" dogmatic who advocates "purist," "barren," "restrictive," "doctrinaire," and "aesthetically paranoid" conceptions of music. ${ }^{2}$ Hanslick's famous essay Vom Musikalisch-Schönen is usually read as an account of music's essential properties that are independent of historical and cultural variation. ${ }^{3}$ Such an essentialist reading is, in turn, easily associated with conservative implications about what counts as good music. Hanslick is portrayed as a purist who, by emphasizing musical rules, puts forth strong restrictions on the practice of music criticism and perhaps even on musical practices themselves. In this sense, musical formalism is taken to be an enemy of artistic freedom. It may also seem that formalism is depriving music of its ability to have moral significance, as the semantic connection to the extramusical is denied by the formalistic view.

Another way of handling musical formalism is the attempt to neutralize it altogether. This is done by claiming that Hanslick's view is not that radical after all. It is not hard to find books or articles suggesting that Hanslick actually endorses some weaker version of the emotionalist theory, i.e., the view that music is in one way or another expressive of human emotions. 
And if not endorsing a weaker version of emotionalist theory, then at least he failed to consider the most viable version of emotionalism. ${ }^{4}$ The claim is that, instead of offering a real alternative to the view that music's content is bound up with human emotions, Hanslick simply criticized nowadays straw-man theories that explain the musical expressiveness of emotions by appealing to composers' self-expression or to the emotions music arouses in the audience. Furthermore, Hanslick is frequently accused of outright inconsistency based on his use of emotive terminology in his musical criticisms. Formalism is such an unsustainable position, it is argued, that even its main advocate could not hold fast to it. ${ }^{5}$ In general, the debate on musical meaning is so dominated by the emotionalist view that discussion on Hanslick typically centers on the question of whether he allowed some kind of relation between music and emotions after all. This is unfortunate as it effectively prevents discussion on what the consequences and the motivations of his account of music as tonally moving forms might be.

In this paper, my goal is to defend musical formalism by placing Hanslick's argument in a broader Kantian framework. Hanslick mentions Kant only once by name in his essay, listing him as one of those "eminent people" who have affirmed the contentlessness of music. ${ }^{6}$ Yet, it is not hard to find fundamentally Kantian elements in Hanslick's work, such as his emphasis on the contemplative and disinterested nature of the aesthetic judgment, the nonconceptuality of music's content, and his insistence that beauty has no purpose. I argue further that these are not merely superficial similarities. In my reading, Hanslick's formalism is in fact motivated by and manifests the Kantian conception of freedom as self-legislation. Thus understood, the only way in which music may be seen as having any moral significance in the first place is by a commitment to the notion of the absolute autonomy of music.

\section{The Autonomy of Musical Beauty}

Hanslick begins his essay by pointing out an error in the musical aesthetics of his day. This error is based on the idea that there is one single essence or quality common to all works of art. According to Hanslick, every other field of aesthetics has abandoned the idea of a general concept of beauty from which the aesthetics of any particular art could be derived. However, musical aesthetics still tries to assimilate music into a paradigm of beauty that explains beauty as being dependent on the content of the artwork. This is how the erroneous thought, identified by Hanslick, works: Paintings are about landscapes or historical events and 
literature is about intriguing characters facing complicated moral dilemmas, so surely music too must be about something. Given that music has no readily perceptible extramusical content, feelings or (in the vocabulary of contemporary musical aesthetics which I shall adopt) emotions must constitute music's content. Consequently, the expression of emotions must be music's primary purpose. ${ }^{7}$

Such is Hanslick's diagnosis of 19th century musical aesthetics. And little has changed. Analytic philosophy of music is dominated by accounts that aim at explaining the relation between music and human emotions. Hanslick rejects this still influential view. According to him, emotions have nothing to do with the content of music. Nor is the arousal of emotions the purpose of music. The arousal of emotions is not the purpose of music, as "beauty has no purpose at all." ${ }^{8}$ Now, this quote might as well be taken from Kant's Critique of the Power of Judgment in which Kant famously argues that the purposiveness we find in a judgment of the beautiful is not based on any real purpose. In Kant's view, judgments of taste have "formal purposiveness" that cannot be subsumed under any concepts. For Kant, a pure judgment of taste is always independent of determinate concepts, ends, or interests, including emotions. ${ }^{9}$ This is also the direction of Hanslick's thought.

According to Hanslick, the musically beautiful is not based on extramusical concepts. This means, first, that even when music (like opera or lied) is composed to a text, music still follows its own specifically musical laws. The more the composer aims at imitating the content of the text, the more the end result approaches musically uninteresting recitative. Hanslick brings up numerous examples to show how texts of different emotive contents may be accompanied by the same musical material without significant loss to the work's aesthetic quality. ${ }^{10}$

Second, as Hanslick among the very first recognizes, emotions are not independent of concepts. Instead, the very definiteness of emotions rests on "ideas, judgments, and (in brief) the whole range of intelligible and rational thought to which people so readily oppose feelings." ${ }^{11}$ Given that music is incapable of denoting concepts, it cannot express, represent, or portray specific emotions. Hanslick grants that music can resemble one aspect of emotions, namely, the motion of the psychological ${ }^{12}$ process: music can be fast, slow, strong, weak, rising, or falling. However, in Hanslick's view, this resemblance is not enough to warrant the interpretation of music as portraying a psychological process of which the same motion is characteristic.

Third, explaining music by reference to composers' intentions would 
also render the musical beauty heteronomous. As Hanslick argues - 100 years before Wimsatt and Beardsley's celebrated article "The Intentional Fallacy" - whatever intentions the composer may have had while composing the work, they have no relevance for the work's meaning. Or more specifically, insofar as such intentions, i.e., musical thoughts, are relevant, they are already embedded in the work itself and hence there is no need to appeal to them in the explanation of the work. ${ }^{13}$ If the composer is dissatisfied with the orchestration of a symphony, say, he does not first turn to investigate his own emotional state to find a solution, as Deryck Cooke would have it. Nor does he consult his general knowledge of the human psyche, as Suzanne Langer suggests. ${ }^{14}$ Instead, the composer treats the problem in terms of musical instruments and their roles in the score. The kinds of problems the composer faces and the kinds of solutions he or she seeks for them arise from music itself, and knowledge of human emotions does not help him or her one bit in tackling such musical problems.

A fourth case of heteronomy lurks in our tendency to describe music in natural language. Contrary to what is often falsely claimed, Hanslick does not ban emotive descriptions of music. ${ }^{15}$ In fact, he grants that sometimes we cannot do without emotive terminology when talking about music. ${ }^{16}$ Nevertheless, given that people often disagree about such descriptions we should be careful not to take such descriptions as evidence of music's content and infer that music depicts, expresses, or refers to something extramusical. According to Peter Kivy, this argument is "very bad." In Kivy's view, "anyone who has ever performed the simple experiment of playing brief expressive passages of the kind of music Hanslick is talking about to a group of quite ordinary listeners [...] knows quite well that the responses, far from being chaotic and random, are actually predictable and consistent." ${ }^{17}$ Now, let me just mention that this has not been my experience. Nor have I not found compelling empirical evidence for such consistency in the listeners' responses beyond the agreement that fast music in major keys may be called "happy" and slow music in minor keys may be called "sad." ${ }^{18}$ But I do not think Kivy's reading is the most charitable rendering of Hanslick's argument either. Hanslick writes:

Who will come forward and venture to declare that some specific feeling is the content of these themes? One person will say "love." Possibly. Another thinks "yearning." Perhaps. A third feels "piety." Nobody can refute any of them. And so it goes. ${ }^{19}$ 
In my reading, Hanslick's point is that emotive descriptions of music lack the kinds of grounds that would force one to accept them as true as well as criteria that could be used to distinguish correct emotive attributions from incorrect ones. Usually there are several, equally apt ways to characterize a given musical theme (and not just in emotive terms but also in many other figurative ways), and there is no point in trying to establish one of such characterizations as the correct description of the theme's content. Hence, instead of reading such descriptions literally, we should settle for assigning the evidentially less demanding status of "figurative" or "metaphorical" to them. ${ }^{20}$

It is also important to notice that Hanslick is not entirely happy with the supposedly more kosher technical analyses either. These, he claims, make "a skeleton out of a flourishing organism"; they are "apt to destroy all beauty but at the same time all misguided interpreting." ${ }^{21}$ This is to say that the more technical descriptions of a given musical passage or work usually amount to nothing but trivialities and as such they leave the rich and concrete auditory reality of music largely untouched. In general the problem is that, insofar as we want to talk about music, we have to use either technical terminology or resort to emotive or other figurative characterizations. In doing so, we are effectively taking a step out of the medium of music itself. This is why Hanslick claims that, in order to convey the content of a musical theme to someone, "we will have to play for him the theme itself."22

The above four ways of subsuming music under extramusical concepts have one problematic thing in common. In all of them, music is explained by appealing to a set of explanans from a realm of phenomena separate from music itself. However - and this, I believe, has been Hanslick's point all along - music has its very own set of laws or rules. Because of this, the attempt to understand music by referring to the laws or regularities of human emotions or forcing music to follow the narrative of a poem is to commit a category mistake. It is like explaining human intentional action in causal terms. In some sense it may be possible. Human action does have a causal aspect to it, composers have mental states that are causally linked to the production of their works, and musical phrases resemble some psychological processes more than they resemble others. But how helpful are such explanations for our understanding of the explanandum? According to Hanslick, not very. It is not just that the appeal to extramusical principles conflicts with the Kantian requirements of nonconceptuality and disinterestedness of the judgment of taste. It is also that "each particular art demands to be 
understood only of itself, through knowledge of its unique technical characteristics." ${ }^{23}$ Only by looking at how the musical elements function in their musical context can we begin to understand the musical reasons behind musical action.

\section{Three Important Points about the Musical Rules}

According to Hanslick, it is the rules of a musical system and nothing else that composers deal with when they write music. Music does not convey extramusical contents that could be translated into another medium. Music's content is the tonal forms themselves. The tonal forms express musical thoughts that are inseparable from the musical forms themselves, and the rules that govern the application of the materials of a musical system reside in the system itself. These rules are also the relevant framework of listening to music with understanding. Does this commit Hanslick to an essentialist view about music as has been assumed by many? I do not think so. Over and over again, Hanslick stresses how, instead of subsuming music under a general metaphysical principle, we should focus on the "immanent" rather than "transcendent" features of music. This means focusing on "music's grammatical rules." ${ }^{24}$

Any mention of rules is obviously likely to strengthen the impression of Hanslick as the pedantic school master caricatured in the role of Beckmesser in Wagner's Meistersinger. And indeed, if the rules Hanslick talks about were independent of the musical system like, e.g., the regulations of the Union of Soviet Composers controlling what passed as politically correct music in the Soviet Union, then the worry would be justified. Regulative rules derived from extramusical principles would indeed limit the freedom of composers as well as turn the understanding of music into an exercise of mechanically translating musical phrases into political dictums or emotional reports. But the musical grammar Hanslick talks about has little to do with such regulative prohibitions and obligations. There is no sanction for failure to follow the musical rules or for breaking them. Rather, the kinds of rules Hanslick refers to constitute the very musical system. One could not compose Western tonal music except by following at least some of those rules.

As Hanslick's choice of the word "immanent" suggests, the musical grammar he has in mind is not like Chomsky's generative grammar that will be revealed some day after years of empirical investigation. Hanslick's primary interest lies in the musical materials themselves, in "the entire system of tones, with their latent possibilities for melodic, harmonic and rhythmic variety." ${ }^{25}$ Insofar as there are any essentialist 
implications here, then they come closer to Wittgenstein's later remark that "essence is expressed by grammar," ${ }^{26}$ which is just another way of saying that there is no essence to be found beyond the conventions of our language or, in Hanslick's case, the conventions of our musical system. In contrast with the spirit of essentialism, Hanslick stresses the historicity and cultural contingency of the musical rules. They do not have a prototype in nature, nor are they created by God. In general, one should "be on guard against the error that this tonal system (this present one) necessarily exists in nature." ${ }^{27}$ Instead, "our Western European music," as Hanslick calls it, is an artifact that has developed gradually and will continue to develop. ${ }^{28}$

It is also noteworthy that, by "tonally moving forms," Hanslick does not primarily refer to large scale forms such as the fugue, the oratorio, or the manifestations of the sonata form as has been assumed by some. ${ }^{29}$ The musical forms Hanslick seems to have in mind are closer to basic principles of harmony, melody, and rhythm. This may strengthen the typical prejudice according to which formalism puts too much emphasis on the structural or syntactic properties of our musical system and does this at the expense of other aspects of music, such as timbre, accentuation, and other such nuances that cannot be captured in the score. But there is no reason why the formalist should assume that musical notation is somehow more authoritative than the actual, performed music. In fact, Hanslick's phrase "tönend bewegte Formen," translated by Payzant as "tonally moving forms," implies that he is talking about music that is already heard and not just studied based on the score. The German word tönend is the adjectival form of the verb tönen, to sound. The movement of the forms, Bewegung der Formen, is thus said to happen by sounding, which implies that the tonal forms are perceived primarily by listening. ${ }^{30}$ This means that also the rules of music are primarily given in the performances of musical works where music's fine nuances are on a par with its more structural properties.

But does not Hanslick's emphasis on the objective rules of music create a gulf between his view and that of Kant? After all, for Kant, it is the subject's feelings of pleasure and displeasure, not the objective rules of art, which function as the determining grounds of a judgment of taste. While it is impossible to fully address the possible disanalogy here, there are two things worth mentioning that might alleviate the worry. First, as I have just argued, Hanslick's rules are not extramusical rule formulations in some written manual for composers. Instead, "they reside, though not in a manner open to scientific investigation, instinctively in every cultivated 
ear, which accordingly perceives the organic, rational coherence of a group of tones, or its absurdity or unnaturalness, by mere contemplation, with no concept as its criterion or tertium comparationis." ${ }^{31}$ In fact, there are no explicit examples of musical rules in Hanslick's essay, but the terms grammar, rule, and technique seem to function as general reminders of the autonomous yet structured medium of music. Second, while for Kant the notion of a rule would typically suggest a connection to a concept, in the context of aesthetics this is not the case. In the few sections of the Third Critique where Kant focuses on art (instead of his main concern, i.e., nature of the judgment of taste), he states that art presupposes rules. The rules of art do not have concepts as their grounds. Nor can they be stated in the form of formulas or precepts. Instead, the rules of art must be abstracted from the products of a genius. The genius, i.e., a particular predisposition of the human mind, cannot itself "describe or indicate scientifically how it brings its products into being," but rather functions as a channel for nature to give the rule to art. While the artistic products of a genius are not the result of imitation, they serve others in that way as standards or rules for judging art. ${ }^{32}$ In the light of these remarks, the potential gulf between Kant and Hanslick does not seem that deep after all.

\section{Rules and Freedom}

Up to now, I have argued that Hanslick is not promoting an essentialist theory of music, rather, he is in the business of defending music's autonomy in a Kantian sense. After all, what would it mean to look for the essence of a cultural phenomenon like music? The essentialist claim would simply be a normative claim in disguise. And this is, in fact, a typical charge against formalism. Hanslick is represented as "a pedantic mediocrity blindly opposing all innovation." 33 The idea is that Hanslick's emphasis on the rules of music entails stylistic preferences and a lack of appreciation for creative freedom and musical progress.

However, rather than seeking to uncover the essence of music or dictate criteria for good music, throughout his essay, Hanslick's focus is on music's content and on musical understanding. ${ }^{34}$ If read in this way, then formalism does not entail the superiority of specific standards for the evaluation of music. Hanslick himself writes:

The whole drift of the present inquiry avoids questions of what ought to be and considers only what is. From this it deduces no particular musical ideal as the only genuine musical beauty but merely establishes in the same way for all schools [of music] what the beautiful is in each, even the most antagonistic. ${ }^{35}$ 
Given that the rules of music are constitutive rather than regulative rules, they do not determine what may be done with them. In actual performances, the rules may be followed faithfully, but they may also be applied in novel ways or broken. It is in the musician's discretion what he or she decides to do with the rules, and perhaps the grounds on which such choices are made may include other things besides the rules themselves. Similarly, the listeners have the freedom to form their own views on performances and works. Just as one can understand a sentence and disagree with what it says, one can recognize the musical goals of a given performance and disagree with the performer's choice to develop this line of performative interpretation as opposed to another.

To be sure, the fact that Hanslick talks about both musical meaning and musical beauty may create the false impression that he is advocating a theory of what counts as good music. However, the formalistic view that music's content resides in the application of the various forms that constitute the tonal system does not entail an explanation of what makes these forms better or worse. Hanslick's point is rather that musical beauty is a special, self-subsistent type of beauty that should not be assimilated into any other type or subsumed under a general concept of beauty. Besides, Hanslick's term "Musikalisch-Schönen” does not mean musical beauty in the evaluative sense of the term. As Geoffrey Payzant, the translator of the standard English edition of Hanslick's essay, has shown, insofar as Hanslick offers a more positive account of this specifically musical type of beauty, it is simply "tonally moving forms." ${ }^{36}$ In other words, the phrase "musically beautiful" is just another expression for the autonomous content of music.

But if there is a normative aspect in Hanslick's essay, then it is related, not to music as such, but to us in our roles as listeners. One of Hanslick's reasons for rejecting the emotionalist view is that it encourages what he calls a "pathological" mode of listening, a mode that turns the listeners' attention away from the concrete features of music itself. It makes no difference for Hanslick whether the listener is looking for emotive satisfaction, better digestion, or moral edification in the musical experience. None of these approaches to music "proceeds out of free self-determination," Hanslick writes. ${ }^{37}$ By contrast, while the formalistic truism of music's content as tonally moving forms does not yet tell us what these forms are or how they are to be performed or developed, it nevertheless encourages the listeners to focus on the music itself. Without attention to the specifically musical, our appreciation of music may turn out to be nothing but an inarticulate and empty exclamation "Ah!", "How charming!" that tells more 
about the subjective states of the listener than about music. Hanslick ridicules such responses, comparing the naive listener to an animal that may be affected by music but does not understand music in a sense relevant for aesthetics. $^{38}$

It is here, I think, that Hanslick comes closest to Kant's philosophy. According to Kant, aesthetic judgments differ in principle from judgments about the agreeable that concern, e.g., the tastes of wine and food. Such culinary judgments are related to desire and to a liking that is conditioned "pathologically" by stimuli. Judgments about the agreeable ultimately refer to empirical generalizations and hence to laws of nature. This is why also non-rational animals are capable of taking pleasure in the agreeable, Kant argues. Yet, aesthetic judgments ought to be qualitatively different. Kant writes: "if our sole aim were enjoyment, it would be foolish to be so scrupulous about the means for getting it." ${ }^{39}$ One might as well enjoy sparkling wine from the Canaries, as Kant suggests. Or light up a cigar, as Hanslick recommends. If one is just looking for emotive satisfaction from music, then it should not matter if the satisfaction results from drugs, piquant delicacies or a hot bath. ${ }^{40}$ In general, Hanslick criticizes the "pathological view" of music for the fact, in that view, "musical achievements are to be included along with products of nature which delight us, but which do not make us think, do not make us aware of the creative intelligence." ${ }^{41}$

Here, too, the point is the nature of the connection between the judgment and its object. For the arousal of emotions by music is a causal process, much like the mechanism between drinking wine and getting drunk. If the listener voluntarily places herself within the causal mechanism of the arousal of emotions, then she also deprives herself of the freedom that music as a system of autonomous rules offers for her contemplation and in that sense for participation. By contrast, according to Kant and - as I have argued - Hanslick, judgments of the beautiful are autonomous in the sense of following their own laws that cannot be translated or reduced to the principles of another medium. An aesthetic explanation that appeals to human emotions overlooks this crucial difference. While the more contemporary versions of the emotionalist account of music may be in many ways more sophisticated than the versions against which Hanslick argues, they do not differ significantly from them in this particular respect. They, too, explain music by reference to an empirical connection between music and human emotions, for example, by reference to a connection resulting from "evolutionary hard-wiring." ${ }^{42}$ If I am right, this kind of a naturalistic solution is simply 
not available for Hanslick. From the Kantian perspective, it is only by reference to autonomous rules as opposed to empirical laws of nature that freedom is conceivable in the first place.

Above, I mentioned Kivy's claim about the listeners' ability to discern music's emotive character, namely, that in his view the listeners' emotive responses are "predictable and consistent." Hanslick, in turn, rejects emotionalism because "the effect of music upon feeling possesses neither the necessity nor the exclusiveness nor the constancy which a phenomenon would have to exhibit in order to be the basis of an aesthetic principle." ${ }^{43}$ In other words, as far as the listeners' emotive responses to music are concerned, Kivy finds them predictable and consistent, Hanslick as lacking exclusiveness and constancy. But it is Hanslick's requirement of necessity that marks the gravest difference between the two. For necessities and evolutionary hard-wiring do not make a good fit. Even if the listeners did, as a matter of empirical fact, agree on what emotive characterizations fit what musical themes, this would not yet be enough for Hanslick. The kind of aesthetic principle he is looking for would rest on a more solid foundation than an empirical generalization ever could even if it were true as a matter of empirical statistical fact.

What, then, is the nature of the necessity Hanslick requires of his ideal principle of musical aesthetics? Payzant compares Hanslick's principle to Kant's categorical imperative. ${ }^{44}$ While I do not share Payzant's view that Hanslick wants to define the essence of music, the kind of principle Hanslick is looking for should indeed be characterized as a transcendental principle. It is meant to have the kind of apodictic force that Kant attributes to his categorical imperative, i.e., that the principle is "bound up with the consciousness of its necessity." ${ }^{\prime 45}$ Kant's categorical imperative or the various formulations of the categorical imperative do not state which acts are right or wrong, but rather aim at capturing something about the basis of morality which any rational being should accept as necessarily true. In my reading, Hanslick's so-called positive thesis that "the content of music is tonally moving forms" is meant to be understood along the same lines. For what would it mean to deny that music is tonally moving forms? For example, the debate between the formalist and the emotionalist does not pertain to the question whether music consists of the movement of tonal forms. To say that it does is as trivial as the claim that language consists of words and sentences. Rather, the debate concerns the further interpretation of the tonal forms. While the formalist holds that the forms do not have any other content than themselves, the emotionalist wants to say 
something more, namely, that the tonal forms are somehow expressive of human emotions and that the understanding of music requires that the listener recognizes emotions in music.

Hanslick rejects the emotionalist theory of music because he sees it as an instance of subjecting music to empirical laws of nature and consequently to heteronomy. The reasons for Hanslick's stance are similar to the reasons for resisting explanations of human action simply by reference to causes; they are related to a concern with giving up an essential aspect of freedom. The only way of protecting the possibility of freedom - freedom in music, freedom in musicianship, freedom in listening to music - is by replacing the heteronomous aesthetic principles with one that arises from music itself. This principle is available in the form of the musical rules. Given that these rules are constitutive of music itself, explaining music by appealing to these rules carries the kind of necessity Hanslick demands of his preferred aesthetic principle of music. ${ }^{46}$

\section{Notes}

1. Eduard Hanslick, On the Musically Beautiful, trans. Geoffrey Payzant (Indianapolis: Hackett, 1986), 29.

2. For example, Allan Janik and Stephen Toulmin, Wittgenstein's Vienna (New York: Simon \& Schuster, 1973), 35, 103-104; Peter Kivy, Sound Sentiment: An Essay on the Musical Emotions (including the complete text of the Corded Shell) (Philadelphia: Temple University Press, 1989), 7, 48; Peter Kivy, The Fine Art of Repetition: Essays in the Philosophy of Music (Cambridge: Cambridge University Press, 1993), 263; Kendall Walton, "Listening with Imagination: Is Music Representational?," Journal of Aesthetics and Art Criticism 52:1 (1994), 47.

3. For example, Peter Kivy, The Fine Art of Repetition, 277; Bela Szabados, "Wittgenstein and Musical Formalism," Philosophy 81: 318 (2006), 651; Robert Yanal, "Hanslick's Third Thesis," British Journal of Aesthetics 46:3 (2006), 263. Unfortunately, even those few exceptional writers who dare defend formalism often choose to do so by speaking of the essence and essential properties of music. See, e.g., Nick Zangwill, "Against Emotion in Music: Hanslick was Right About Music," British Journal of Aesthetics 44: 1 (2004); Geoffrey Payzant in Eduard Hanslick, On the Musically Beautiful, Introduction.

4. For example, Peter Kivy, The Fine Art of Repetition, 279; Robert Yanal, "Hanslick's Third Thesis," 260-261.

5. For example, Robert W. Hall, "On Hanslick's Supposed Formalism in Music," Journal of Aesthetics and Art Criticism 25, no. 4 (1967), 433-436.

6. Eduard Hanslick, On the Musically Beautiful, 77. 
7. Ibid., $1-2$.

8. Ibid., 3 .

9. Immanuel Kant, The Critique of the Power of Judgment, trans. Paul Guyer and Eric Matthews, ed. Paul Guyer (Cambridge: Cambridge University Press, 2000), $\iint 11-16$.

10. Eduard Hanslick, On the Musically Beautiful, 21-27.

11. Ibid., 9. Later, this idea has been made famous especially by Ludwig Wittgenstein and his followers who have pointed out the dependence of emotions on their linguistic and behavioral context. See Ludwig Wittgenstein, Philosophical Investigations, trans. G. E. M. Anscombe (Oxford: Blackwell, 1953); Anthony Kenny, Action, Emotion and Will (London: Routledge, 1963).

12. In the Payzant translation the sentence in question reads as: "It can reproduce the motion of a physical process...". This is a slip. In the original German text the word is "psychischen," i.e., "psychological." See Eduard Hanslick, Vom Musikalisch-Schönen (Leipzig : Breitkopf und Härtel, 1854/1874), 16.

13. "We do not look for representations of specific mental processes or events in music, but above all else for music itself [...]. In music no amount of 'intention' can replace 'invention.' Whatever does not become outwardly apparent is, so far as music is concerned, altogether nonexistent, but what has become outwardly apparent has ceased to be mere intention." (Eduard Hanslick, On the Musically Beautiful, 36.)

14. Deryck Cooke, The Language of Music (Oxford: Oxford University Press, 1959), ch. 4; Susanne Langer, Philosophy in a New Key (Cambridge, Mass.: Harvard University Press, 1942), 221.

15. See, for example, Bela Szabados, "Wittgenstein and Musical Formalism," 651,653 .

16. Eduard Hanslick, On the Musically Beautiful, 32.

17. Peter Kivy, The Fine Art of Repetition, 281-282.

18. For a comprehensive list of empirical studies, see Alf Gabrielsson and Patrick N. Juslin, "Emotional Expression in Music," Handbook of Affective Sciences (Oxford: Oxford University Press, 2003), 509-514.

19vEduard Hanslick, On the Musically Beautiful, 14.

2o. Ibid., 32.

21. Ibid., 14 .

22. Ibid., 81 .

23. Ibid., 2.

24. Ibid., 31, 2.

25. Ibid., 28.

26. Ludwig Wittgenstein, Philosophical Investigations, $\mathbb{3} 37$.

27. Eduard Hanslick, On the Musically Beautiful, 70. 
28. Ibid., 69-70.

29. See, for example, Bela Szabados, "Wittgenstein and Musical Formalism," 655 .

30. True, it is possible for one to "listen to music" in one's imagination, e.g., when studying the score, but this possibility rests on the listener's previous experiences of listening to musical performances. One cannot imagine, e.g., the timbre of the cello without previous perceptions of it.

31. Eduard Hanslick, On the Musically Beautiful, 31.

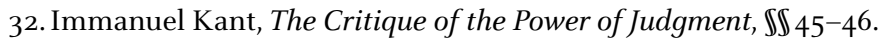

33. Allan Janik and Stephen Toulmin, Wittgenstein's Vienna' 103.

34. A look at Appendix B of Hanslick's essay supports this reading. In the appendix, Hanslick gives numerous quotes from writers who, in his view, advocate what he calls the feeling theory of music, and his examples are of theories that deal primarily with musical meaning (Eduard Hanslick' On the Musically Beautiful, 86-102).

35. Ibid., 38.

36. Geoffrey Payzant's Introduction in Eduard Hanslick, On the Musically Beautiful, 93-94. Hanslick writes: "What kind of beauty is the beauty of a musical composition? It is a specifically musical kind of beauty. By this we understand a beauty that is self-contained and in no need of content from outside itself, that consists simply and solely of tones and their artistic combination." (Ibid., 28.)

37. Ibid., 61.

38. Eduard Hanslick, On the Musically Beautiful, 62.

39. Immanuel Kant, The Critique of the Power of Judgment, 5:208, $\iiint_{4-5}$.

40. Eduard Hanslick, On the Musically Beautiful, 59, 62; Immanuel Kant, The Critique of the Power of Judgment, 5:212; see also Ludwig Wittgenstein, The Blue and Brown Books (Oxford: Blackwell, 1958), 178.

41. Eduard Hanslick, On the Musically Beautiful, 60.

42. See Peter Kivy, Sound Sentiment, 83, 172.

43. Eduard Hanslick, On the Musically Beautiful, 7.

44. Geoffrey Payzant's Introduction to Eduard Hanslick, On the Musically Beautiful, xiv.

45. Immanuel Kant, Critique of Pure Reason, trans. and ed. Paul Guyer and Allen W. Wood (Cambridge: Cambridge University Press, 1998), B41.

46. I would like to thank Risto Pitkänen, Karsten Harries, Lars-Olof Åhlberg, Jonathan Neufeld, as well as an anonymous referee for their insightful comments on earlier versions of this paper. Thanks are also due to the Academy of Finland for financial support of my work. 